\title{
Participant Training for a Flight Test Evaluation of Interval Management
}

\author{
Roy Roper ${ }^{1}$, Brian Baxley ${ }^{1}$, Kurt Swieringa ${ }^{1}$, Clay Hubbs ${ }^{2}$ \\ ${ }^{1}$ National Aeronautics and Space Administration, Langley Research Center, Hampton, VA, \\ United States of America \\ roy.d.roper@nasa.gov, brian.t.baxley@nasa.gov, kurt.a.swieringa@nasa.gov \\ ${ }^{2}$ All Aspect Aerospace Innovations, LLC., Parker, CO, United States of America \\ clay.hubbs@aaainnovate.com
}

\begin{abstract}
Interval Management is a concept designed to be used by air traffic controllers and flight crews to more efficiently and precisely manage inter-aircraft spacing. NASA, in cooperation with Boeing, Honeywell, and United Airlines, tested an avionics prototype onboard flight test aircraft. A critical need was identified to train the pilots participating in the flight test prior to the first flight. This paper documents the flight training regimen that successfully trained the pilots on the Interval Management concepts and flight crew procedures and suggests potential improvements to future training regimens for industry use.
\end{abstract}

Keywords: Interval Management · Pilot training · Cockpit procedures · Flight test

\section{Introduction}

On January 1, 2020, the Federal Aviation Administration (FAA) mandate for Automatic Dependent Surveillance-Broadcast (ADS-B) equipage on aircraft operating within most U.S. airspace will take effect. Those operators equipped to meet the requirements of 14 CFR 91.225 and 91.227 will broadcast aircraft state information, allowing a receiver unit onboard an aircraft to utilize information from other participating aircraft. The capstone flight test of the NASA Air Traffic Management Technology Demonstration (ATD-1) conducted in central and eastern Washington State in early 2017 used ADSB data to perform Interval Management (IM) spacing operations with three aircraft. Speed control directed by a Flight Deck-based IM (FIM) avionics prototype allowed pilots to achieve or maintain a precise time or distance behind a preceding aircraft. In order to test the FIM avionics prototype, a United Boeing 737-900 (B737), Honeywell Boeing 757-200 (B757), and Honeywell Falcon 900 conducted numerous arrival operations into Grant County Airport, WA.

The ATD-1 research activity consisted of three NASA developed technologies expected for the Next Generation Air Transportation System, or NextGen, airspace environment. Two of the components were ground-based for air traffic control (ATC), 
consisting of the Controller-Managed Spacing decision support tool and the Traffic Management Advisor and Terminal Metering Scheduler [1, 2, 3]. Both enable increased use of performance-based navigation procedures. The third component, FIM, was hosted onboard an aircraft and allowed pilots of that vehicle to set a specific time or distance interval (e.g., 94 seconds spacing or 12 NM spacing) from a preceding aircraft on the same or different route, conforming to that goal by a point common to both aircraft. To test the robustness of the system, the flight test examined how well the algorithm managed to reduce the error from a predetermined time or distance goal. For example, if two aircraft were separated by 150 seconds and the spacing goal was set at 180 seconds, one could see how well the algorithm eliminated the 30 seconds of error. The first aircraft was the Target aircraft, while the second vehicle, known as the Ownship, performed the IM operation. In this example, the spacing algorithm calculates an appropriate slower speed based on the position of the two aircraft and arrival procedures they are flying to slow the FIM-equipped Ownship aircraft and create 30 seconds of additional space between the two vehicles. For the flight test, two pairs of vehicles were used to observe intermeshed IM operations. The first pair was the Falcon 900 typically followed by the B757. The second pair was the same B757 followed by the B737. Both the B757 and the B737 were similarly equipped and could execute IM operations, and therefore could be interchanged as needed between the second and third positions.

There were two fundamental goals of the training: flight crew procedures to conduct the operation, and correct aircraft positioning when setting up each scenario. The first goal was to give the pilot experience with the prototype avionics to correctly and efficiently enter information, and also manage the aircraft's airspeed and vertical path while conducting the IM operation. The intent of this goal was to reduce the distraction from normal flight duties and minimize the impact to pilot workload during high-task periods. Simulation was used to allow pilots to practice operating the FIM avionics. The participating pilots, using unfamiliar multi-step software for a new airspace procedure, faced considerable learning and operability hurdles to achieve proficiency. During the training activity, the test pilots had to familiarize themselves with a new airspace, the FIM prototype, and the flight test procedures which included four types of IM clearances [4].

For the second goal, since the ground-based components were not evaluated as part of this flight test, the pilots, flight test director (FTD), and ATC had to work together to precisely and efficiently position all three aircraft for each test run. The aircraft had to be correctly positioned so that the spacing interval between the Target and Ownship aircraft resulted in the desired spacing error, a process which ultimately required two stages of refinement. Multiple test runs were planned for each flight test day with each aircraft potentially moving to a new start position after each run, meaning travel lengths from airport to start position would vary from run to run. Correct coordination was required to minimize inefficiencies due to time spent positioning aircraft. During the development process, a further need was identified - for the pilots and FTD to train together as part of an integrated team. The FTD was expected to be the coordinator for aircraft positioning and would be alongside NASA experts during the flight test. Due to the complex and challenging nature of positioning the aircraft for the next scenario while also entering the FIM data, the crew and FTD were trained as a team with special emphasis on Crew Resource Management specific to the flight test. The communication 
used between pilots and the flight test director greatly simplified the management of duties and created a realistic atmosphere from which both could train for the flight test.

\section{Background}

In order to ensure development of the proper skillset, NASA created a product that engaged the learner in a simple-to-learn and satisfying way. Research by De Cino [5] states, "Complex technology systems that are difficult to master and use, often create problems of usability for system users. User experience and satisfaction with the technology is partly a function of the learnability of the system, and directly affects the student during the training process.” Since every person learns differently, a diversified stepwise training regimen was developed as the best means to educate and ensure transfer of knowledge for the majority of pilots. Each step was designed to train data entry and operation of the software in some incremental way while maintaining an effective learning experience. Research by Bell and Koslowski [6] describes several key features of learning which were desirable training attributes for both this activity and future experiments.

\section{Training Methodology}

As stated previously, a multi-tiered, incremental approach was used to prepare the pilots participating in the flight test for the next, more complex phase of training. This training regimen consisted of three steps: computer-based training (CBT), classroom training, and simulator training. Since the acquisition of basic concepts results from repeated exposure, the FIM prototype display was included in every learning exercise. The computer-based training and user guide for the prototype was initially provided to participants ahead of training at NASA Langley Research Center (LaRC). It contained reading material, video, and was implemented with a guided touch-screen interface. Later instruction occurred in a classroom setting using interactive training. Then fully immersive training was given using flight simulators to conduct simulated scenarios in the flight test airspace. This was followed by extensive debriefs where problems and irregularities were discussed and corrected.

Prior to training the pilots participating in the flight test, two separate groups of four pilots each were recruited for development of the training program and scenarios flown in the simulators. Each group consisted of two crews qualified to fly the B737 or B757. Each development crew was made up of a pilot with previous FIM research experience and one without. The groups were each staggered by two weeks for two sessions, allowing researchers to observe learning retention over a one-month period between each test group's session. This was intentional since the pilots participating in the actual flight test were expected to have approximately one to two months between the simulation training and the flight test. When brought back after one month, all developmental pilots needed retraining and simulator practice sessions to reach similar levels of proficiency as the previous session. These pilots did not have access to the CBT since it was in development at the time, and for some, it took nearly the full week to get back up to speed. 
Pilots participating in the flight test were selected by their respective flight operations departments and included Honeywell flight test pilots, United flight test pilots, and United line pilots. All of the pilots were current and qualified to fly the aircraft in the position(s) they flew during the flight test. Each had glass cockpit experience from at least two different manufacturers, were Required Navigation Performance (RNP) qualified, and had a minimum of 50 hours of flight experience for the year prior to selection. The pilots ranged in age from 42 to 69 with an average age of 53, and had a diverse background in terms of hours, qualifications, and experience with flight tests. The pilots had each been flying from 20 to 49 years with total flight time accruals from 4,500 to 13,000 hours. Nine pilots were selected and trained, and eight of them flew in the flight test itself. Boeing provided two FTDs who were also a part of the group training sessions. Both were familiar with the airspace used during the flight test, and one was a former air traffic control manager for the test area.

The pilots were specifically required to learn how to operate the prototype FIM software and operational techniques of the aircraft to conduct IM operations. Both pilots and flight test directors needed to learn how to coordinate the setup of flight vehicles prior to initiating a test run.

The procedure used by the pilots and FTD to set up the beginning of each scenario was as follows:

1. Using the aircraft's Flight Management Computer (FMC) estimated time of arrival function, each flight crew gave the FTD their respective time to a waypoint specified for that scenario.

2. The FTD then assigned specific scheduled time of arrivals (STAs) to each aircraft separated by three minutes (e.g., 0901, 0904, 0907 Coordinated Universal Time).

3. Each crew then managed their speed and ground track to achieve the specified STA prior to initiating the IM operation.

4. Once the IM operation was initiated, the spacing interval calculated by the prototype FIM avionics was displayed on the electronic flight bag (EFB), which the flight crew then communicated to the FTD.

5. The FTD assigned a new spacing goal based on the spacing interval previously given by the flight crew plus the spacing error defined in the test matrix for that scenario.

6. The flight crew reentered the FIM information with the new assigned spacing goal, then flew the operation by setting the FIM commanded speed in the mode control panel speed window.

The procedure described herein is particular to the flight test in order to achieve specific test criterion. In future operations with the ATD-1 concept fully deployed, ATC will issue a spacing goal and the pilot might not have knowledge of the error. The pilot will simply follow speed commands output by the algorithm to the conclusion of the IM operation. A breakdown by training modality is presented in the following sections. 


\subsection{Computer-Based Training}

The CBT was developed using Adobe Captivate 9 software. Many airlines provide electronic learning to pilots frequently on travel via their electronic devices (typically an iPad) to allow anywhere/anytime training. The pilot gains knowledge via reading, video, and quizzes outside of the classroom, and alleviates some of the scheduling and financial burden for the airline by reducing overall time needed with an instructor at the training center. It has been demonstrated that prior training on a PC-based flight simulation package, regardless of the method to manipulate the flight controls, resulted in better overall performance than an untrained operator [7]. Dennis and Harris go on to describe, "...the best performance was observed in the group of participants that had prior simulation training using a representative set of flight controls to interact with..." The NASA LaRC CBT was designed to provide a walk-through of the prototype FIM avionics, and allow the user to learn the layout of the system. The difference from most contemporary software is that NASA provided a manipulatable product to enhance ab initio flight training rather than static displays. This part-task CBT was the pilots' initial training on the FIM prototype system and took approximately 80 minutes to complete. The CBT was later supplemented with classroom academic instruction and training in high-fidelity simulators.

The CBT allowed the users to conduct training at their convenience, could be repeated as often as desired, and contained no pass/fail pre-qualification, enabling the user to make mistakes without fear of negative repercussions. Guided instruction was provided before most button presses in beginning modules. Later modules followed similar button pathways without instruction, forcing the learner to actively develop the cognitive template for the required task, and only provided guided instruction when new information was encountered.

The CBT was as close to the expected FIM prototype as possible (see Fig. 1 compared to [8]). Sized to the Electronic Flight Bag (EFB), the product looked dimensionally similar when viewed on an iPad and operated much like the FIM prototype to be used for the flight test, therefore creating a realistic product that moved at the learning pace of the trainee. Training on a similar device allowed the pilots to practice task requirements.

Supplemental materials appended to the CBT included charts describing IM published procedures and a pilot guide which describes IM clearance types with associated graphics of each operation using a Standard Terminal Arrival Route (STAR), legends for all FIM displays, and descriptions of functionality for every button in the EFB.

The CBT chapters included: 1) CBT Info - How to operate the CBT, 2) Introduction - General overview of IM and primary aircraft components, 3) Ownship Entry - How to input Ownship information into the EFB, 4) Clearances - How to input Target information into the EFB by clearance type, and 5) Operation - Air crew procedures and operational techniques once engaged in IM.

The CBT discussed the flight test airspace, special STARs for the flight test, specific definition and description of four IM clearance types, a walkthrough of the FIM prototype Human-Machine Interface (HMI), and the flight crew procedures.

Pilots participating in the development phase did not have access to the CBT as the pilots participating in the flight test did. As a result, it was clear to the researchers 
that the pilots who had access to the CBT prior to arriving at NASA LaRC needed much less training time to learn EFB functionality due to the effectiveness of the CBT. Therefore, the CBT and all supporting materials remained available to the flight test participants during the interim period between training and actual flight with a brief one-hour refresher the day prior to the flight test.

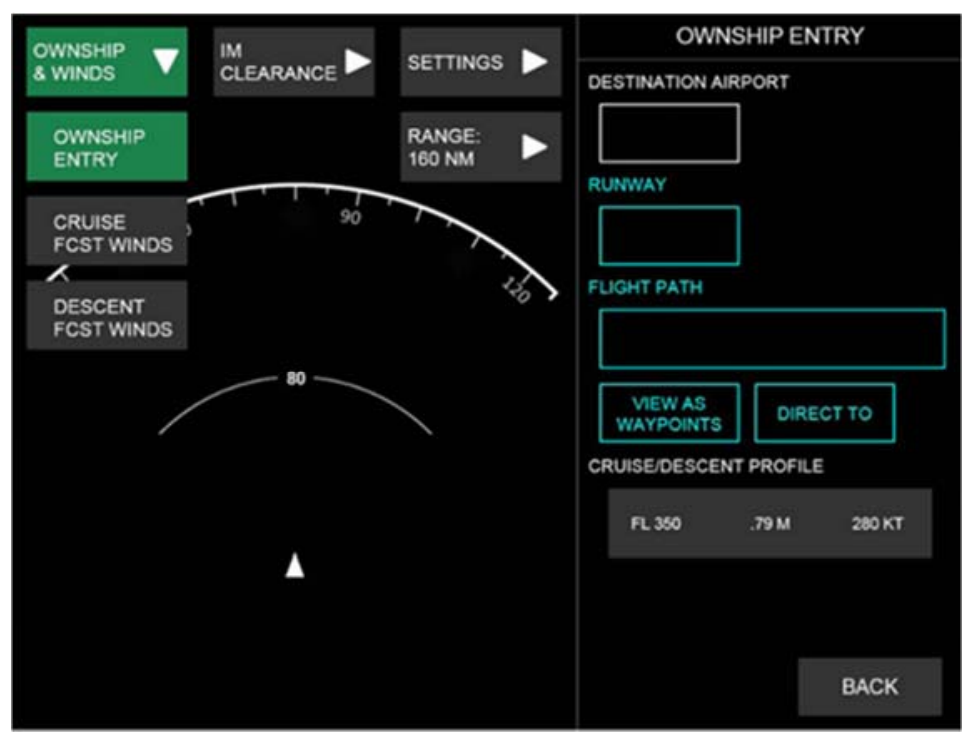

Fig. 1. CBT entry page example

\subsection{Classroom Instruction}

Classroom instruction further defined pilot roles and actions to accomplish the scenario setup and conduct the IM operation. The instruction at NASA LaRC was designed to provide graduated daily training to coincide with the simulator regimen. One and a half hours on the first day and one hour on the second day was dedicated to training. Focus was on Interval Management clearance types, walk-through review of EFB functionality, flight crew procedures, flight test card formats, and methods of managing the aircraft's energy to conform to the vertical path and FIM speed commands. The third and fourth day focused on crew and FTD integration, to include the FTD giving the morning briefing for the scenarios to be simulation flown that day, and answering questions from the pilots. Attendees included one FTD, one B737 flight crew, one B757 flight crew, instructor, and researchers associated with the learning task for the day. All remaining time during the four-day training session was spent in simulator training (described below). Each training day concluded with debriefing sessions to clarify irregularities and solidify lessons learned.

Interactive classroom instruction was employed to permit defining the flight crew procedures and information on the test cards. Classroom instruction reiterated training from the CBT. Immediately prior to the flight test, and approximately six weeks after training at NASA LaRC, two one-hour classroom sessions of training and review 
were given to the pilots. This consisted of a short walk-through of the prototype FIM avionics, aircraft operations while performing IM, and a question and answer period.

\subsection{Simulator Training}

The NASA LaRC B757 and B737 simulators were the primary locations for practicing EFB manipulation similar to the prototype and understanding user interactions between the prototype and IM operations. Simulator training occurred over four days to develop operational experience (including data entry on a labor intensive prototype), confidence, and scan technique with the added FIM displays. Following the classroom instruction on the first day, the flight crews initially went to their respective simulator for familiarization and part-task training of the IM procedure. Afterward, the two simulators and FTD desktop station were connected together to simulate as realistically as possible the scenarios to be flown during the flight test, with a pre-recorded Target serving as the lead aircraft in all cases. During the flight test itself, the flight test director was expected to operate from within one of the test aircraft using voice communications to disseminate information to the pilots of all three aircraft. Therefore, during training at NASA LaRC, the FTD was staged separately at a desktop simulator station to emulate the FTD flight station during the real-world test. The distributed simulation provided audio connectivity and Cockpit Display of Traffic Information between all simulators and also included simulated traffic similar to typical traffic in the planned flight test location.

In stand-alone mode, the training in the simulators provided the pilots the opportunity to familiarize themselves with data entry into the FIM prototype and practice the pilot procedures to conduct a IM operation. The integrated simulations with both flight crews and the FTD provided the opportunity for all participants to practice the procedures to conduct multiple IM operations during a single flight and understand the pacing for data entry. Trainers allowed both pilots and the FTD to make mistakes, and gave positive encouragement for successful planning and execution. Instructors narrated a run summary prior to each start, emphasizing specific pitfalls to recognize and avoid, including an explanation of the FIM algorithm operation. The algorithm is a closed-loop system whereby errors or delays in implementing the commanded FIM speed simply result in issuance of a new speed command when the original command is not followed. Once this was known, the pilot fixated less on the speed command window and focused on the operation in general; in effect, reducing pilot workload and overall stress when attempting to adhere to the assigned task.

Exploratory learning was guided during initial simulator training, and then a 'hands off' approach was used during later lessons while instructors added realism to the simulation by providing radio inputs as ATC and other traffic. Quick reference guides were provided to manage programming the EFB, if needed. Data cards were also developed detailing key information specific to each run. The flight test consisted of 38 test conditions, where each aircraft had a separate start condition based on the run, resulting in 114 data cards (38 conditions x 3 aircraft). For training, a smaller set of runs was selected that represented the majority of conditions expected for the flight test. The scenario setup design flowed from simple clearance types to complex, which progressively increased pilot workload. 
The pilots and FTD also received operational procedure training to accurately pre-position aircraft on the appropriate STAR for that scenario to within three minutes of goal times prior to executing an experimental run. The FTD was specifically given extra opportunities for exploratory learning to develop the correct mental model of aircraft positions by testing various time intervals between aircraft and start locations to see what worked best. The FTD was able to develop correction strategies to manage cases where the initial spacing error was too far out of range. In some cases, towards the end of the training program, the NASA instructor left the training area during critical phases of decision-making and remotely observed the FTD and pilot interactions to ensure the scenarios were being executed as desired.

\section{$4 \quad$ Effectiveness of Training Program}

Critical components of the training regimen which led to the success of the flight test were pilot understanding of Interval Management, the development of flight test cards for the pilots, setting time goals to ensure correct aircraft positioning, and establishing constructive interactions between flight test crew members. After the conclusion of the flight test, six of eight test pilots completed a survey about the effectiveness of each learning method and any need for improvement. During the flight test, the Ownship used a spacing interval of either time or distance to determine separation from the Target aircraft. Pilot actions to conduct both operations were identical in either case. Numerical analysis of time-based operations is used below to provide an empirical example of learning transfer since these were the majority of flight test results. More detailed analysis of the flight test can be found in [9]. The success of the training is discussed next in terms of pilot feedback of the CBT, classroom training, knowledge transport in simulation training, and operational effectiveness during the actual flight test.

\subsection{CBT}

The majority of pilots indicated their needs were met by the CBT through repetitive physical programming and overview of IM clearance types. Following the flight test, pilots suggested additional modules desired for future CBTs should include contingencies to FIM abnormalities and an instructional video of data entry prior to the interactive portion. The authors believe a video showing entry for each IM clearance type would be beneficial to the learner. The CBT and flight simulator EFB were created during different development periods of the prototype FIM software, leading to slight differences in practice devices. Several responses stated the need for identical training products to prevent confusion during training. The pilots also reported that the CBT would have benefited from a clearer description of the FIM avionics prototype, in particular the displays and messages shown to the pilots. They also stated a defined correlation between the Target aircraft's behavior and its impact on the IM operation would be relevant to future FIM pilots. 


\subsection{Classroom Training}

The majority of pilot responses to the training survey stated classroom time devoted to FIM should be minimal to none, indicating higher knowledge transport from the CBT and flight simulator training for the majority of pilots. The maximum classroom time desired by pilot respondents in future training was two hours. However, from the instructor's perspective, two pilots benefitted more readily from the classroom setting than the CBT due to highly interactive conversations. Classroom debriefs following simulator training were invaluable to pilot understanding.

\subsection{Simulator Training}

During flight simulator training, researchers observed seven of nine flight test pilot trainees with no Interval Management flight experience were able to adequately program the EFB and follow most speed commands by the end of the first day $(<4$ simulation hours). All were able to do so by the end of the second training day ( $<7$ total simulation hours). When asked, "How many hours of simulator time do you think it took before you felt comfortable performing Interval Management following a Target aircraft?”, the average response was four hours. Pilots suggested IM integration with line operation training would be preferred over stand-alone IM training to minimize simulation training time. One pilot commented simulator training time and value would be optimized if multiple, short scenarios focused on EFB programming, stopping once FIM guidance is initialized. Subsequent simulation training could concentrate on operational techniques while FIM is active. When asked, "What percentage of simulation time was spent learning how to program the EFB?”, pilot perceptions ranged from $20 \%$ to $80 \%$ with an average of $58.75 \%$. While the sample size is very small, this does indicate the FIM avionics prototype required a high mental workload as currently implemented. Some pilots believe an overall reduction of button presses for subsequent prototypes will reduce training time. Unless changed, a quick reference guide would be "extremely helpful” in the training environment. Respondents noted that simulation time could be minimized if FIM and RNP approach training were integrated, since both are expected NextGen products and could be accomplished using similar training tactics already in place.

\subsection{Operational Effectiveness}

The pilots were able to accomplish 157 flight test runs over 19 days [9, 10]. Though exceeding the desired goal of 124 runs, not all runs fit within the planned test matrix. Initial flights of the prototype software revealed critical anomalies allowing only a few runs per day. Correct coordination between the FTD and pilots ensured initial start times were relatively accurate, but some runs were filtered from the data set due to the anomalies. As the underlying issues were corrected and pilot and FTD experience increased, the number of planned runs increased to seven per day. A total of 129 time based runs were recorded with FIM spacing goals ranging from 124 to 300 seconds and a mean spacing interval of 178 seconds. Of the 118 time-based operations deemed feasible for evaluation at the final approach fix (FAF), the mean error from spacing goal at the FAF (i.e., completion point) was approximately two seconds [9]. This equates to 
within $1 \%$ of the mean desired spacing interval, indicating the pilots were able to execute the procedures they learned during training to conduct the IM operation and achieve the desired spacing goal.

Of the 129 time-based operations, 106 required the pilot to enter an assigned spacing goal, while for the remaining 23 time-based operations the spacing goal was calculated by the IM avionics prototype to maintain the current spacing between aircraft. The desired range of correction was from 60 seconds early to 60 seconds late with increments of $0,15,20,30$, and 60 seconds in both directions ( -60 to +60 seconds). Across all analyzed runs, the absolute value of the average difference between the initial spacing errors the pilots were trying to achieve and actually achieved was 28 seconds. The training and procedures described in Section III was one impact to this metric, however it was also potentially impacted by differences between aircraft flight management systems and the difference between the forecast winds and actual winds. Training strategies employed by the FTD to mitigate instances where aircraft positioned outside the desired range also influenced this metric.

As a measure of learning transfer, we can observe how trained versus untrained crews positioned aircraft prior to each run. The crews onboard two of the three flight test aircraft had undergone simulator training at NASA LaRC to practice the operational setup of aircraft prior to the start of a run. Of runs analyzed, 57 run setups involved both Ownship and Target crews which had trained, while the remaining 49 contained one crew without training. During the flight test, the crew of every aircraft acted independently using holds and speed inputs to meet the STA given by the FTD by the start of the run. Considering 60 seconds as the maximum deviation for initial spacing error, the setups with wholly trained crews achieved this criteria $95 \%$ of their attempts, while the setups with one untrained crew achieved this criterion in $73 \%$ of their attempts. Additional information was unavailable to conduct a more complete analysis, however, the fact that crews trained at Langley consistently positioned their aircraft closer to the conditions on the test card compared to the crews not trained at Langley is suggestive of the effectiveness of the training program. Over the course of 19 flight days, the deviation from the desired initial spacing error did not appreciably improve for either type, indicating improvement was not a function of training, but potentially due to the tools available to the flight test participants attempting to position the aircraft as ATC would in the NextGen environment.

During the time-based runs analyzed, the pilot responded to new commanded speeds an average of 10.6 times per run, which corresponded to 0.57 speed changes per minute of operation using the mode control panel to enter a speed command [11]. The pilot took an average of 8.51 seconds to input each new speed command with dispersion of 5.8 seconds. Based on previous research $[12,13]$ and researchers' observation during the training regimen and flight test, the authors speculate there was strong retention of knowledge from training. The flight crew typically programmed all variables to the EFB correctly, allowing active engagement of the FIM system, and responded to speed commands in a timely manner as trained, leading to a high degree of accuracy for the operation. 


\section{$5 \quad$ Future Training}

In the future, IM operations may become the norm, therefore requiring less extensive training for understanding the use of the procedures. It would be expected that initial IM information from the FAA would be followed by training materials and CBT products developed by individual airlines and training vendors. Within the airline industry, CBT can maximize pilot availability, allow the airline to track student understanding and time spent on each subject area, and can register whether the learner has completed coursework. Using CBT, the pilot can preview the subject material ahead of instructor guided classroom learning. A fully functional version of the system interface can permit the user to gain operable experience through exploratory learning, and testing the interface. The classroom experience can be maximized by using that time to reiterate and interactively test working knowledge with an expert instructor, thereby developing user confidence and trust in the system. Regarding future FIM flight simulation training, pilot comments suggest introducing ATC communications across multiple controller handoffs to create additional realism for better transfer of knowledge. Pilots stated that understanding the algorithm behind FIM was not required for training, but specific procedures for Ownship programming and Target selection must be trained.

Based on the experience of the flight test, the close timeframe of all three activities, CBT, classroom training, and flight simulator training, resulted in a cohesive program that was satisfactory and relevant for all participants. After completion of such a program, pilot responses indicated access to a CBT should be a minimal requirement for the interim period between training and later flight use to refresh skills as needed. Continued access to learning materials following the program would help promote continuity of training.

\section{Summary}

Prior to conducting a flight test of a prototype FIM system for ATD-1, NASA realized pilots and FTD training prior to the first flight was critical due to the unique and complex nature of the software and the operations themselves. A learning regimen was devised that incorporated CBT, classroom instruction, and distributed live simulator training. All three phases were interactive and occurred over a short timeframe to allow learners to progress from rudimentary understanding of the concept and software, to manipulation and control of the aircraft, to setting up the scenarios and flying the IM operation. Key features found to be successful in assisting the pilots to correctly set up the scenarios and conduct the IM operation included repetitive physical programming to learn the system, CBT to minimize or make classroom time more constructive for the learner, integrated training with other team members, and progressive training of more realistic and complex simulation scenarios. The results of the ATD-1 flight test training regimen offer strong evidence that a fully functional CBT enabled the pilots and FTD to have effective exploratory learning prior to the classroom instruction and simulator training. The integrated simulator training allowed the pilots and FTD to practice conducting the IM operations in a live and interactive manner, which proved to be essential during the flight test itself to maximize the number of scenarios flown each 
day. The short duration between the three training methods, and the short duration between the conclusion of the training regimen and the beginning of the flight test, contributed significantly to the overall effectiveness of the ATD-1 training program.

\section{References}

1. Robinson III, J.E., Thipphavong, J., Johnson, W.C.: Enabling Performance-based Navigation Arrivals: Development and Simulation Testing of Terminal Sequencing and Spacing system. Air Tfc. Ctrl. Qtly., vol. 23, no. 1, pp. 5--27 (2015)

2. Callantine, T.J., Hunt, S.M., Prevot, T.: Simulation Evaluation of Controller-Managed Spacing Tools Under Realistic Operational Conditions. HCI-Aero '14 Proc. Intl. Conf. HumanComp. Interact. Aerosp., New York, NY, Assoc. Comp. Mach. (2014)

3. Barmore, B., et al.: Interval Management: Development and Implementation of an Airborne Spacing Concept. AIAA Guidance, Navigation, and Control Conference, AIAA 2016-1608, San Diego, CA (2016)

4. Van Tulder, P.A.: Flight Deck Interval Management Flight Test Final Report. NASA CR2017-219626 (2017)

5. De Cino, T.J.: A Usability and Learnability Case Study of Glass Flight Deck Interfaces and Pilot Interactions Through Scenario-based Training. Nova Southeastern University (2016)

6. Bell, B.S., Kozlowski, S.W.J.: Active Learning: Effects of Core Training Design Elements on Self-Regulatory Processes, Learning, and Adaptability. J. Appl. Psy., vol. 93, no. 2, pp. 296--316 (2008)

7. Dennis, K.A., Harris, D.: Computer-based Simulation as an Adjunct to Ab-initio Flight Training. Intl. J. Avn. Psy., vol. 8, no. 3, pp. 261--276 (1998)

8. Alves, E.: FIM Avionics Operations Manual. NASA CR-2017-219593 (2017)

9. Swieringa, K., et al.: Flight Test Evaluation of the ATD-1 Interval Management Application. 17th AIAA Aviation Technology, Integration, and Operations Conference (ATIO), AIAA 2017-4094, Denver, CO (2017)

10. Baxley, B.A., et al.: Flight Crew Survey Responses from the Interval Management (IM) Avionics Phase 2 Flight Test. 17th AIAA Aviation Technology, Integration, and Operations Conference (ATIO), AIAA 2017-4095, Denver, CO (2017)

11. Scharl, J.: Boeing Post-Flight Data Analysis Report. The Boeing Company, Seattle, WA, doc. num. D780-10416-1 (2017)

12. Baxley, B., et al.: Human-in-the-loop Assessment of Alternative Clearances in Interval Management Arrival Operations. NASA TP-2016-219362 (2016)

13. Baxley, B., et. al.: Experiment Description and Results for Arrival Operations using Interval Management with Spacing to Parallel Dependent Runways. NASA TP-2013-217998 (2013) 